\title{
Tauroursodeoxycholic acid protects bile acid homeostasis under inflammatory conditions and dampens Crohn's disease-like ileitis
}

\author{
Lien Van den Bossche, Daniel Borsboom, Sarah Devriese, Sophie Van Welden, Tom Holvoet, Lindsey Devisscher, \\ Pieter Hindryckx, Martine De Vos and Debby Laukens
}

Bile acids regulate the expression of intestinal bile acid transporters and are natural ligands for nuclear receptors controlling inflammation. Accumulating evidence suggests that signaling through these receptors is impaired in inflammatory bowel disease. We investigated whether tauroursodeoxycholic acid (TUDCA), a secondary bile acid with cytoprotective properties, regulates ileal nuclear receptor and bile acid transporter expression and assessed its therapeutic potential in an experimental model of Crohn's disease (CD). Gene expression of the nuclear receptors farnesoid X receptor, pregnane $\mathrm{X}$ receptor and vitamin $\mathrm{D}$ receptor and the bile acid transporters apical sodium-dependent bile acid transporter and organic solute transporter $\alpha$ and $\beta$ was analyzed in Caco-2 cell monolayers exposed to tumor necrosis factor (TNF)a, in ileal tissue of TNF ${ }^{\triangle A R E / W T}$ mice and in inflamed ileal biopsies from $C D$ patients by quantitative real-time polymerase chain reaction. TNF ${ }^{\triangle A R E / W T}$ mice and wild-type littermates were treated with TUDCA or placebo for 11 weeks and ileal histopathology and expression of the aforementioned genes were determined. Exposing Caco-2 cell monolayers to TNFa impaired the mRNA expression of nuclear receptors and bile acid transporters, whereas co-incubation with TUDCA antagonized their downregulation. TNF ${ }^{\triangle A R E / W T}$ mice displayed altered ileal bile acid homeostasis that mimicked the situation in human CD ileitis. Administration of TUDCA attenuated ileitis and alleviated the downregulation of nuclear receptors and bile acid transporters in these mice. These results show that TUDCA protects bile acid homeostasis under inflammatory conditions and suppresses CD-like ileitis. Together with previous observations showing similar efficacy in experimental colitis, we conclude that TUDCA could be a promising therapeutic agent for inflammatory bowel disease, warranting a clinical trial.

Laboratory Investigation (2017) 97, 519-529; doi:10.1038/labinvest.2017.6; published online 6 February 2017

Inflammatory bowel diseases (IBD), including Crohn's disease (CD) and ulcerative colitis (UC), are chronic relapsing disorders of the gastrointestinal tract with unknown etiology. Increasing evidence suggests that the reduced abundance and richness of the gut microbiota plays a pivotal role in the pathogenesis of IBD. ${ }^{1}$ Interestingly, reduced microbial enzymatic activity in the gut lumen of IBD patients results in bile acid dysmetabolism, which is characterized by defective bile acid deconjugation, desulphation and transformation to secondary bile acids. Because some of the secondary bile acids produced by intestinal bacteria exert anti-inflammatory effects on gut epithelial cells, intestinal bile acid dysmetabolism significantly contributes to the pathogenesis and symptoms of IBD. ${ }^{2}$

Bile acids are cholesterol derivatives that act as signaling molecules by activating nuclear receptors and G-protein- coupled receptors. Activation of these receptors alters the expression of genes involved in different processes, including bile acid homeostasis, lipid metabolism and inflammation. ${ }^{3,4}$ The immunomodulatory roles of bile acids have been thoroughly investigated over the last years. Several studies indicated a role for the bile acid-activated nuclear receptors farnesoid X receptor (FXR), pregnane X receptor (PXR) and vitamin $\mathrm{D}$ receptor (VDR) in the immunoregulation induced by bile acids in innate immune cells and gut epithelial cells. ${ }^{5-8}$ Moreover, selective FXR, PXR or VDR agonists reduce the inflammatory response in experimental models of intestinal inflammation, ${ }^{5,6,8-10}$ whereas mice deficient for one of these receptors are sensitized to gut inflammation. ${ }^{5,9,10}$ In addition, $\mathrm{FXR}^{-1-}$ and $\mathrm{PXR}^{-/-}$mice exhibit a compromised epithelial barrier, ${ }^{10,11}$ and spontaneously develop colitis or ileitis. ${ }^{5,10,12}$ 
Thus, changes in nuclear receptor signaling severely affect the course of intestinal inflammation.

Several lines of evidence support the possibility that nuclear receptor signaling is impaired in IBD patients. First, genetic variations in genes encoding PXR, FXR and VDR have been associated with IBD. ${ }^{13-19}$ Furthermore, mRNA expression levels of FXR and VDR are reduced in inflamed intestinal mucosa from IBD patients, ${ }^{20}$ whereas $P X R$ expression is downregulated in inflamed ileum of pediatric $\mathrm{CD}$ and in both inflamed and noninflamed colon from active UC patients. ${ }^{20-22}$ Also, the apical sodium-dependent bile acid transporter $(A S B T)$ that mediates bile acid uptake across the brush-border membrane of ileal enterocytes, ${ }^{23}$ is downregulated in inflamed ileum of CD patients. ${ }^{20,24}$ This finding can be indirectly related to impaired bile acid signaling through nuclear receptors, since disturbed intestinal bile acid transport results in aberrant intracellular bile acid levels. ${ }^{25}$ Importantly, $A S B T$ gene expression is also reduced in noninflamed ileal tissue from CD patients in remission and UC patients with active colonic disease, ${ }^{20,24}$ showing that these molecular changes are not per se directly associated with inflammation. However, inflammatory mediators have the potential to negatively regulate the expression of nuclear receptors and bile acid transporters. ${ }^{26-31}$ Proinflammatory cytokine expression therefore induces a vicious cycle leading to the perpetuation of the intestinal inflammation. Hence, normalizing the expression of intestinal bile acid transporters and/or nuclear receptors could be useful to alleviate chronic inflammation in IBD.

Selected bile acid species have been shown to regulate the expression of intestinal bile acid transporters. ${ }^{32-36}$ One of the bile acids that has received much attention over the last years is tauroursodeoxycholic acid (TUDCA). TUDCA is a secondary bile acid with cytoprotective properties ${ }^{37}$ that ameliorates colonic inflammation in mice. ${ }^{38-40}$ However, as bile acids are primarily reabsorbed in the terminal part of the small intestine, with only a small proportion $(<5 \%)$ of the intestinal bile acid pool passing into the colon, ${ }^{23}$ it is more reasonable to study bile acid supplementation in a model of ileal inflammation. In this study, we investigated whether TUDCA alleviates the disruption of ileal bile acid homeostasis upon tumor necrosis factor (TNF) $\alpha$ stimulation and evaluated whether this bile acid improves CD-like ileitis in mice.

\section{MATERIALS AND METHODS}

\section{Caco-2 Cell Culture}

Caco-2 cells (HTB-37, ATCC Cell Biology Collection, Manassas, VA, USA) were seeded on 24-well semipermeable inserts $(0.4 \mu \mathrm{m}$, translucent ThinCerts, Greiner Bio-One, Vilvoorde, Belgium) at a density of $10^{5}$ cells per well and cultured for 2 weeks in Dulbecco's modified Eagle medium supplemented with $10 \%$ heat-inactivated fetal calf serum and $10 \mathrm{mM}$ HEPES buffer (all Life Technologies, Ghent, Belgium). Developing Caco-2 monolayer integrity was monitored by measuring transepithelial electrical resistance
(TEER) using a Millicell ERS-2 Voltohmmeter (Merck Millipore, Billerica, MA, USA). Two weeks post seeding, when TEER values of $\sim 700 \Omega . \mathrm{cm}^{2}$ were obtained, the fully differentiated Caco- 2 monolayers were incubated basolaterally with $100 \mathrm{ng} / \mathrm{ml}$ recombinant human TNF $\alpha$ (Life Technologies) or an equal volume of medium, and apically with 250 or $500 \mu \mathrm{M}$ TUDCA (Prodotti Chimici e Alimentari S.p.A., Italy) or an equal volume of medium. Each condition was performed in triplicate. After $48 \mathrm{~h}$, the TEER of the Caco2 monolayers was measured, cells were collected and total RNA was isolated for quantitative real-time polymerase chain reaction (PCR). Medium from the basolateral compartment was used to determine the concentration of interleukin (IL)-8.

\section{Patient Characteristics and Sample Collection}

Mucosal biopsy specimens from actively inflamed areas of terminal ileum of $\mathrm{CD}$ patients were sampled during endoscopy. As a control group, mucosal samples from the terminal ileum of healthy individuals were included. CD was diagnosed based on clinical, endoscopic and histological criteria. All CD patients underwent endoscopy to determine the severity of mucosal inflammation. In healthy individuals, endoscopy was performed for reasons other than IBD (screening for colorectal cancer, abdominal pain or rectal bleeding). In all healthy subjects, the ileum appeared normal during endoscopy, which was confirmed by histopathology. Patients characteristics are shown in Table 1. All biopsy specimens obtained during endoscopy were immediately placed in RNAlater and stored at $-80^{\circ} \mathrm{C}$.

\section{Administration of TUDCA to TNF ${ }^{\triangle A R E / W T}$ Mice}

C57BL/6 J TNF ${ }^{\triangle A R E / W T}$ mice were kindly provided by $\mathrm{Dr}$ George Kollias (Alexander Fleming Biomedical Sciences Research Center, Vari, Greece). Starting at 4 weeks of age, male $\mathrm{TNF}^{\triangle \mathrm{ARE} / \mathrm{WT}}$ mice $(N=11)$ and wild-type littermates $(N=5)$ were given TUDCA (Calbiochem, Germany) in the drinking water at a concentration of $2 \mathrm{~g} / \mathrm{l}$ until the end of the study. Placebo-treated TNF ${ }^{\triangle \mathrm{ARE} / \mathrm{WT}}$ mice $(N=7)$ and wildtype littermates $(N=8)$ received normal drinking water during the entire study period. All animals had free access to food and water. Body weight was monitored twice a week and mice were killed by cervical dislocation at 15 weeks of age. The distal ileum was removed and flushed with phosphate-buffered saline (PBS). Fragments of $5 \mathrm{~mm}$ were cut and samples were immersed in $4 \%$ formaldehyde (Klinipath, Olen, Belgium) or RNAlater (Ambion, Cambridgeshire, UK).

\section{RNA Extraction}

Total RNA was extracted from Caco-2 cells and human and mouse distal ileal samples using the Qiagen RNeasy Mini Kit (Qiagen, Venlo, The Netherlands) with on-column DNase treatment. Concentration and purity of the total RNA was determined using nanodrop technology (BioPhotometer Plus, 
Table 1 Patient characteristics

\begin{tabular}{|c|c|c|}
\hline & $C D$ & Healthy control \\
\hline N & 10 & 10 \\
\hline Gender (male/female) & $5 / 5$ & $2 / 8$ \\
\hline Age (years, mean (range)) & $28.7(21-38)$ & $46.4(23-67)$ \\
\hline Age at diagnosis (years, mean (range)) & $25.6(17-38)$ & - \\
\hline Disease location (L1/L3) & $6 / 4$ & - \\
\hline \multicolumn{3}{|l|}{ Medication } \\
\hline No & 7 & 10 \\
\hline Corticosteroids & 2 & - \\
\hline Questran & 1 & - \\
\hline \multicolumn{3}{|l|}{ Surgical history } \\
\hline Ileal resection & 2 & - \\
\hline Appendectomy & 1 & - \\
\hline Hemicolectomy & 2 & - \\
\hline Cholecystectomy & 1 & - \\
\hline \multicolumn{3}{|l|}{ Indication for endoscopy other than IBD } \\
\hline Abdominal pain & - & 2 \\
\hline Screening for polyps or cancer & - & 6 \\
\hline Occult blood in feces & - & 1 \\
\hline Gross rectal bleeding & - & 1 \\
\hline
\end{tabular}

Eppendorf, Rotselaar, Belgium). All samples exhibited an OD260/OD280 ratio between 1.8 and 2.1.

\section{Quantitative Real-Time PCR}

One microgram of total RNA was converted to single-stranded cDNA by reverse transcription using the SensiFAST cDNA Synthesis Kit (Bioline Reagents, UK) according to the manufacturer's instructions. The cDNA was diluted to a concentration of $5 \mathrm{ng} / \mu \mathrm{l}$ and $15 \mathrm{ng}$ was used in real-time PCR with SYBR Green (SensiMix SYBR No-ROX Kit, Bioline Reagents) and $250 \mathrm{nM}$ of each primer (BioLegio, Nijmegen, The Netherlands). A two-step program was performed on the LightCycler 480 (Roche, Belgium). Cycling conditions were $95^{\circ} \mathrm{C}$ for $10 \mathrm{~min}, 45$ cycles of $95^{\circ} \mathrm{C}$ for $10 \mathrm{~s}$, and $60^{\circ} \mathrm{C}$ for $1 \mathrm{~min}$. Melting curve analysis confirmed primer specificities. All reactions were performed in duplicate. Expression data were calculated relative to the mean of the overall expression level and normalized to the stably expressed housekeeping genes hydroxymethyl-bilane synthase (HMBS), succinate dehydrogenase complex A subunit (SDHA) and tyrosine 3-monooxygenase/tryptophan 5-monooxygenase activation protein zeta isoform (YWHAZ) for the Caco-2 cells, to glyceraldehyde-3-phosphate dehydrogenase (GAPDH), HMBS, hypoxanthine-guanine phosphoribosyltransferase (HPRT) and SDHA for the human ileal biopsy samples, and to Gapdh, Hmbs and Sdha for mouse ileal tissue. The PCR efficiency of each primer pair was calculated using a standard curve of reference cDNA. Amplification efficiency was determined using the formula $10^{-1 / \text { slope }}$. Sequences of the primer sets and the PCR efficiencies are listed in Table 2. Gene expression levels are expressed as normalized relative quantities (NRQ).

\section{Luminex}

IL-8 secretion by Caco-2 cells into the basolateral medium was measured using the Bio-Plex Pro Human Cytokine Assay (Bio-Rad), according to the manufacturer's protocol. Measurements were performed using the Bio-Plex MAGPIX Multiplex Reader and data were analyzed with the Bio-Plex Manager 6.1 software (Bio-Rad).

\section{Histological Assessment of Intestinal Pathology}

Ileal tissue sections of $4 \mu \mathrm{m}$ were stained with hematoxylin and eosin and scored in a blinded fashion. Histological sections were evaluated for villous destruction and bowel wall influx of inflammatory cells. Villous destruction was scored on a scale of $0-3: 0$, normal; 1 , thickened villi; 2 , blunted villi; 3 , destructed villi. Bowel wall infiltration was scored using the following scoring system: 0 , normal; 1 , infiltrate into muscular layer of mucosa; 2 , infiltrate into submucosa with sporadic granulomas; 3 , infiltrate through submucosa into muscularis propria (and/or confluent granulomas); 4, regional transmural infiltration; 5, diffuse transmural infiltration and/or crypt abcedation. The sum of the individual components was expressed as the total inflammation score.

\section{Statistical Analysis}

Statistical analysis was performed using SPSS Statistics version 22.0 (IBM SPSS, Chicago, USA) and GraphPad Prism version 4 (GraphPad, California, USA). NRQ values were logtransformed for statistical analysis. All data are expressed as mean \pm s.e.m. Body weight changes were analyzed using linear mixed models. Data were tested for normality using the Kolmogorov-Smirnov test. Comparisons between two groups were performed using the unpaired Student's $t$-test for normally distributed data, applying the Welch's correction in case of unequal variances, or the Mann-Whitney $U$-test for non-normally distributed data. Statistical analysis for multiple comparisons was performed using one-way analysis of variance (ANOVA), with subsequent Tukey or GamesHowell post hoc tests, depending on the homogeneity of variances. Two-tailed probabilities were calculated and $P$-values of $\leq 0.05$ were considered statistically significant. Multivariate analysis was carried out using SIMCA version 14.0 (Umetrics, Umea, Sweden). Principal component analysis was executed on the gene expression data of nuclear receptors and bile acid transporters in $\mathrm{TNF}^{\triangle \mathrm{ARE} / \mathrm{WT}}$ mice.

\section{Ethical Considerations}

The use of patient material was approved by the Ethics Committee of the Ghent University Hospital (permit number UZG 2004/242) and all patients provided written informed 
Table 2 Primers sequences for quantitative real-time PCR analysis

\begin{tabular}{|c|c|c|c|c|}
\hline Gene symbol & Species & Forw $\left(5^{\prime}-3^{\prime}\right)$ & $\operatorname{Rev}\left(5^{\prime}-3^{\prime}\right)$ & $E(\%)$ \\
\hline Asbt & Mouse & CCCAAATGCAACTGTCTGCG & CACCCCATAGAAAACARCACCA & 102 \\
\hline Fxr & Mouse & CGGCAGGCAGAATAAAAGGG & GTGAGCGCGTTGTAGTGGT & 101 \\
\hline Gapdh & Mouse & CATGGCCTTCCGTGTTCCTA & GCGGCACGTCAGATCCA & 87 \\
\hline Hmbs & Mouse & AAGGGCTITTCTGAGGCACC & AGTTGCCCATCTITCATCACTG & 95 \\
\hline Osta & Mouse & TCTGCACCCACGGTGGTAT & GGCCATTTCTACAAGTGTGAGG & 98 \\
\hline Ost $\beta$ & Mouse & AGATGCGGCTCCTTGGAATTA & TGGCTGCTITCGATTTCTG & 103 \\
\hline Pxr & Mouse & GATGGAGGTCTTCAAATCTGCC & CAGCCGGACATTGCGTTC & 98 \\
\hline Sdha & Mouse & CTTGAATGAGGCTGACTGTG & ATCACATAAGCTGGTCCTGT & 103 \\
\hline$V d r$ & Mouse & GTGCAGCGTAAGCGAGAGAT & GGATGGCGATAATGTGCTGTTG & 100 \\
\hline$A S B T$ & Human & GGACAATGCAACAGTITGCTC & CCGTACTTAGGACCACACTTAGG & 101 \\
\hline FXR & Human & GACTITGGACCATGAAGACCAG & GCCCAGACGGAAGTTTCTTATT & 101 \\
\hline GAPDH & Human & TGCACCACCAACTGCTTAGC & GGCATGGACTGTGGTCATGAG & 91 \\
\hline HMBS & Human & GGCAATGCGGCTGCAA & GGGTACCCACGCGAATCAC & 101 \\
\hline HPRT & Human & TGACACTGGCAAAACAATGCA & GGTCCTIITCACCAGCAAGCT & 103 \\
\hline OSTa & Human & TCATTTCCCGTCAAGCCAGG & GGCGAACAAGCAATCTGCC & 95 \\
\hline OST $\beta$ & Human & TCCAGGCAAGCAGAAAAGAAA & ACTGACAGCACATCTCTCTCT & 99 \\
\hline$P X R$ & Human & TTGCCCATCGAGGACCAGAT & GTCTCCGCGTTGAACACTGT & 93 \\
\hline SDHA & Human & TGGGAACAAGAGGGCATCTG & CCACCACTGCATCAAATTCATG & 92 \\
\hline$V D R$ & Human & TCTCCAATCTGGATCTGAGTGAA & GGATGCTGTAACTGACCAGGT & 101 \\
\hline YWHAZ & Human & ACTTTTGGTACATTGTGGCTTCAA & CCGCCAGGACAAACCAGTAT & 93 \\
\hline
\end{tabular}

consent. The mice were housed in the laboratory animal facility at Ghent University Hospital according to the institutional animal healthcare guidelines. This study was approved by the Institutional Review Board of the Faculty of Medicine and Health Sciences of Ghent University (ECD2014-25).

\section{Results}

TNFa-Induced Downregulation of Nuclear Receptors and Bile Acid Transporters in Caco-2 Cell Monolayers is Antagonized by TUDCA

It is well known that the expression of nuclear receptors and bile acid transporters is directly controlled by inflammatory cytokines. ${ }^{26-31}$ We initially examined the effect of TNF $\alpha$ on the mRNA expression of the nuclear receptors FXR, PXR and $V D R$ and the bile acid transporters $A S B T$, organic solute transporter (OST) $\alpha$ and OST $\beta$ in Caco- 2 cell monolayers. The addition of $100 \mathrm{ng} / \mathrm{ml} \mathrm{TNF} \alpha$ to Caco- 2 cells for $48 \mathrm{~h}$ resulted in a reduced expression of $P X R(P=0.029$; Figure 1a), while there was a non-significant trend toward a downregulation of FXR and $\operatorname{VDR}(P=0.127$ and $P=0.057$, respectively; Figure 1a). Concerning the bile acid transporter genes, TNF $\alpha$ reduced gene expression levels of ASBT, OST $\alpha$ and OST $\beta$, but this decrease was not statistically significant for $A S B T$ and OST $\alpha(P=0.271$ and $P=0.071$, respectively; Figure $1 \mathrm{~b})$.
Because previous studies reported a role for bile acids in the regulation of intestinal bile acid transporter expression, ${ }^{32-36}$ we next determined if TUDCA could inhibit the TNF $\alpha$ induced downregulation of the aforementioned genes. The addition of 250 or $500 \mu \mathrm{M}$ TUDCA to the apical compartment of the Caco- 2 cell monolayers prevented the TNF $\alpha$ mediated repression of $P X R$ and OST $\alpha$. In addition, FXR and $A S B T$ gene expression tended to increase when $500 \mu \mathrm{M}$ TUDCA was added to the apical compartment $(P=0.075$ and $P=0.073$, respectively). However, decreased mRNA levels of $V D R$ and OST $\beta$ remained unaffected upon TUDCA co-incubation (Figures $1 \mathrm{a}$ and $\mathrm{b}$ ). In order to evaluate whether the observed effects are associated with a possible anti-inflammatory effect of TUDCA, we measured IL-8 secretion into the basolateral medium. This cytokine is highly responsive to TNF $\alpha$ stimulation via nuclear factor-kappa $B$ $(\mathrm{NF}-\kappa \mathrm{B})$-mediated mechanisms and is most commonly used as a readout of anti-inflammatory efficacy in Caco-2 cells. $^{2,41-43}$ Incubating cells with $100 \mathrm{ng} / \mathrm{ml}$ TNF $\alpha$ for $48 \mathrm{~h}$ resulted in a significant induction of IL-8 secretion, which was not prevented by the addition of 250 or $500 \mu \mathrm{M}$ TUDCA to the apical compartment (Figure 1c). Furthermore, since $\mathrm{TNF} \alpha$ increases tight junction permeability through NF- $\kappa \mathrm{B}$ activation, ${ }^{44}$ monolayer integrity was assessed by TEER measurements as another indirect measure of NF- $\kappa \mathrm{B}$ activity. 
a

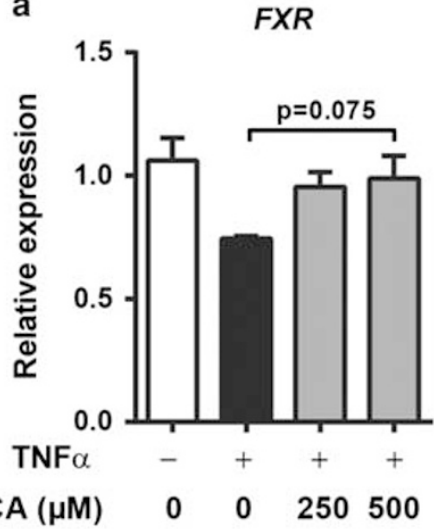

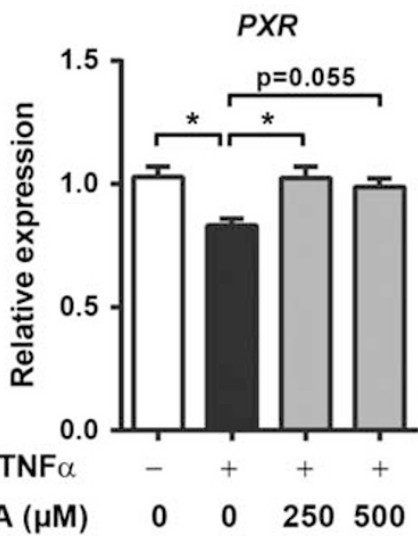

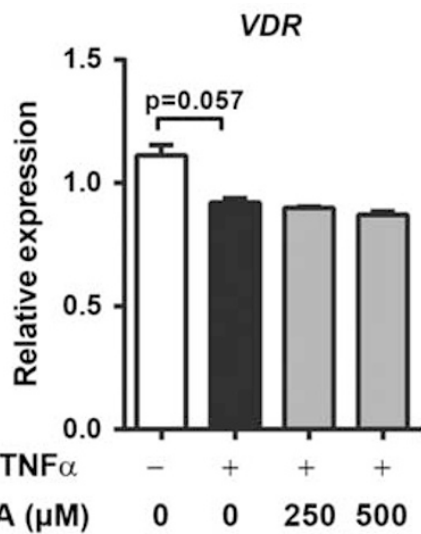

b
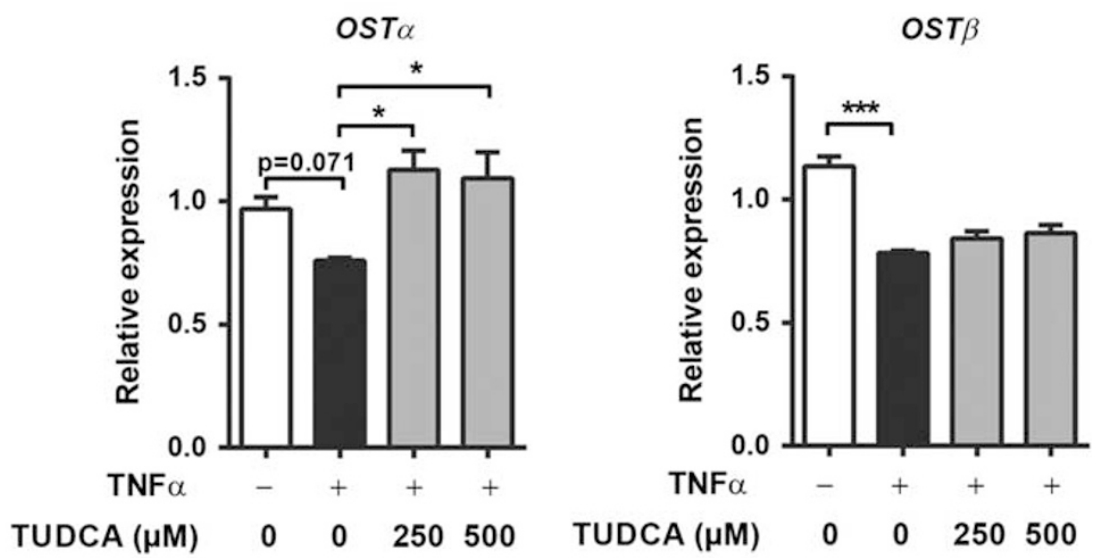

$\operatorname{TUDCA}(\mu \mathrm{M})$

$0 \quad 0 \quad 250500$

$\operatorname{TUDCA}(\mu \mathrm{M})$

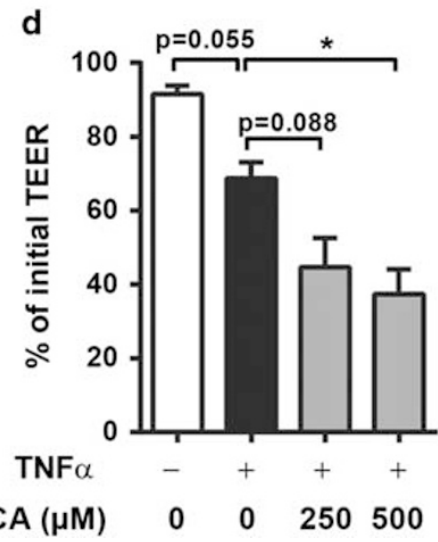

Figure 1 Effect of TUDCA incubation on TNFa-induced repression of genes involved in bile acid homeostasis. Caco-2 cell monolayers were simultaneously exposed to $100 \mathrm{ng} / \mathrm{ml}$ TNFa (basolateral) and 250 or $500 \mu \mathrm{M}$ TUDCA (apical) for $48 \mathrm{~h}$. (a) Normalized mRNA expression levels of the bile acid-activated nuclear receptors. (b) Normalized mRNA expression levels of the main bile acid transporters. (c) Secretion of IL-8 into the basolateral medium. (d) TEER expressed as a percentage of the initial corresponding TEER values (prior to experiment). Data are represented as the mean \pm s.e.m. from three replicates in one experiment. Gene expression data were log-transformed. ${ }^{*} P \leq 0.05,{ }^{* *} P<0.01,{ }^{* * *} P<0.001$.

Exposing Caco-2 cell monolayers to TNF $\alpha$ alone for $48 \mathrm{~h}$ reduced TEER ( $P=0.055$ compared to control monolayers), which decreased even more when 250 or $500 \mu \mathrm{M}$ TUDCA was co-administered apically $(P=0.088$ and $P=0.033$ compared to TNF $\alpha$ alone, respectively; Figure 1d). However, TUDCA was not toxic to Caco-2 cells as assessed by lactate dehydrogenase release (Supplementary Figure S1). These data indicate that TUDCA protects from disruption of bile acid homeostasis upon $\mathrm{TNF} \alpha$ stimulation via mechanisms that are likely independent from any anti-inflammatory effect.

\section{$\mathrm{TNF}^{\Delta \mathrm{ARE} / \mathrm{WT}}$ Mice Display Altered lleal Bile Acid Homeostasis Resembling Human CD lleitis}

Because excessive TNF $\alpha$ production drives the onset of ileitis in $\mathrm{TNF}^{\triangle \mathrm{ARE} / \mathrm{WT}}$ mice, ${ }^{45}$ which is believed to closely mimic human CD ileitis, ${ }^{45,46}$ we determined whether this model 
carries the aberrant gene expression signatures related to intestinal bile acid homeostasis in $\mathrm{CD}$. Therefore, gene expression levels of nuclear receptors and bile acid transporters were determined in ileal tissue of 15-week-old $\mathrm{TNF}^{\triangle \mathrm{ARE} / \mathrm{WT}}$ mice (when ileal inflammation is fully established ${ }^{46}$ ) and wild-type littermates, as well as in ileal tissue of CD patients with active ileal disease (L1 and L3) and healthy subjects. Patient characteristics are shown in Table 1. Regarding the nuclear receptors (Figure 2a), ileal FXR expression was decreased by $43 \%$ in CD patients $(P=0.019$, compared to controls) and by $50 \%$ in $\mathrm{TNF}^{\Delta \mathrm{ARE} / \mathrm{WT}}$ mice $(P<0.001$, compared to wild-type mice). In addition, we also observed a decrease in mRNA levels of PXR and VDR in the inflamed ileum of both $\mathrm{CD}$ patients and $\mathrm{TNF}^{\mathrm{ARE} / \mathrm{WT}}$ mice.
Gene expression analysis of ileal bile acid transporters (Figure 2b) revealed that $A S B T$ was significantly downregulated in CD ileitis (40\% of control values, $P=0.018$ ). Similarly, ileitis in $\mathrm{TNF}^{\triangle \mathrm{ARE} / \mathrm{WT}}$ mice was associated with a marked decrease in gene expression of the main bile acid uptake transporter Asbt (22\% of control values, $P<0.001$ ). Although CD patients did not exhibit significant differences in the expression levels of the basolateral efflux transporters OST $\alpha$ and OST $\beta$, these transporters were significantly reduced in $\mathrm{TNF}^{\triangle \mathrm{ARE} / \mathrm{WT}}$ mice as compared to their wild-type littermates $(P<0.001$ and $P=0.035$, respectively; Figure $2 \mathrm{~b})$. These results show that $\mathrm{TNF}^{\triangle \mathrm{ARE} / \mathrm{WT}}$ mice exhibit major changes in ileal bile acid homeostasis that closely mimic those observed in human CD ileitis.
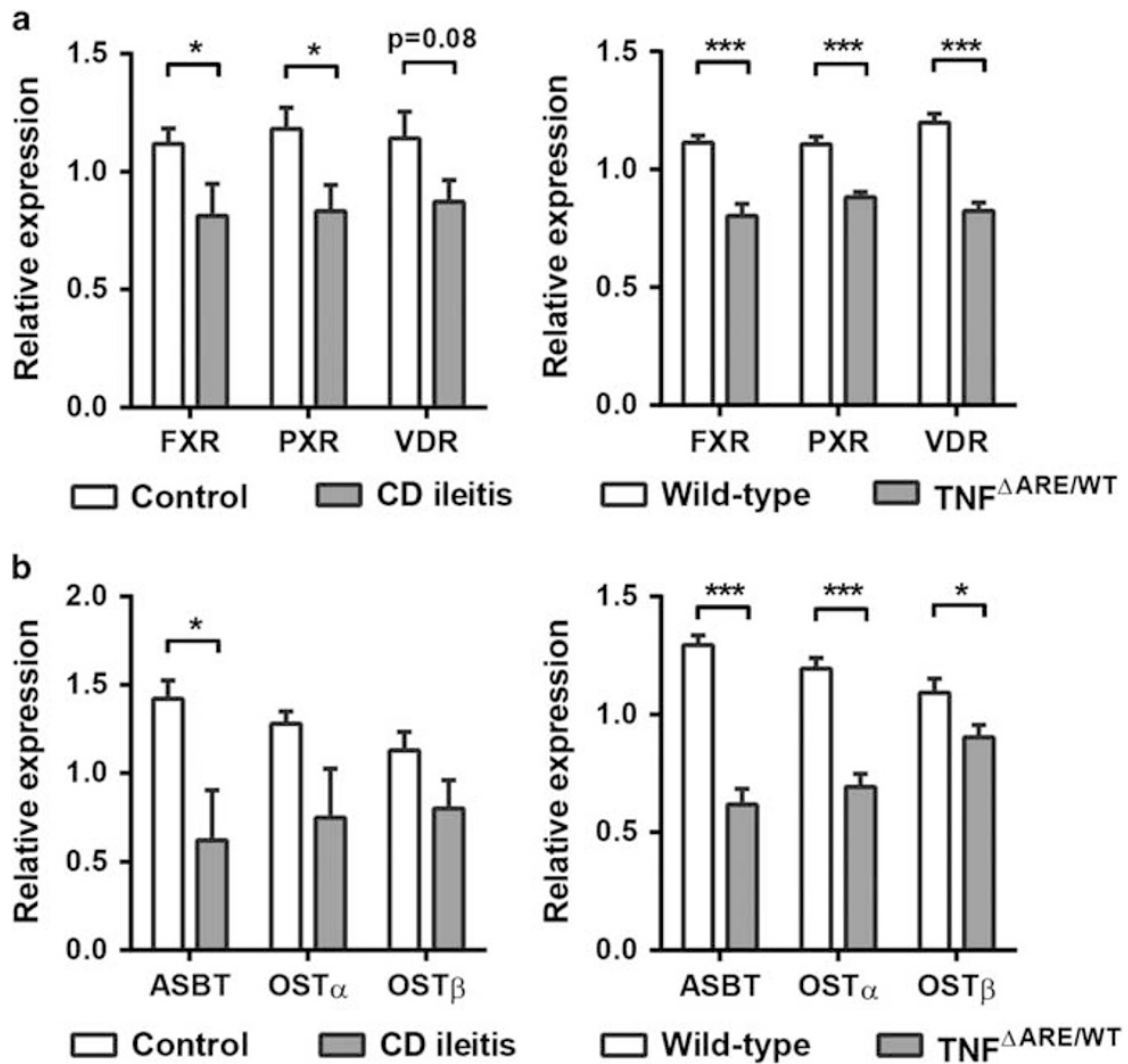

Figure 2 Expression of genes involved in ileal bile acid homeostasis in CD patients and TNF ${ }^{\triangle A R E / W T}$ mice. Normalized mRNA expression levels of bile acid-activated nuclear receptors (a) and the main bile acid transporters (b) in ileal biopsies from healthy controls and CD patients (left, $N=10$ in each group) and 15-week-old TNF ${ }^{\triangle A R E / W T}$ mice and wild-type littermates (right, $N \geq 7$ in each group). Data are represented as the mean \pm s.e.m. Gene expression data were log-transformed. ${ }^{*} P \leq 0.05,{ }^{* * *} P<0.001$.

Figure 3 Effect of TUDCA administration on the expression of genes involved in ileal bile acid homeostasis. Normalized mRNA expression levels of the bile acid-activated nuclear receptors (a) and the main bile acid transporters (b) in ileal tissue of 15-week-old TNF ${ }^{\triangle A R E / W T}$ mice and wild-type (WT)

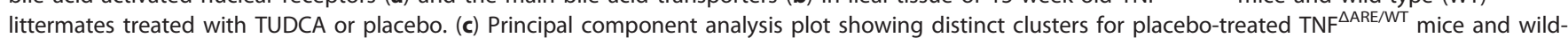
type littermates. Each mouse is represented as a point: circles for wild-type mice and triangles for TNF ${ }^{\triangle A R E / W T}$ mice. The filled symbols represent TUDCAtreated mice, whereas placebo-treated mice are shown by open symbols. Notice the shift of TNF ${ }^{\triangle A R E} / \mathrm{WT}$ mice toward wild-type mice after prolonged treatment with TUDCA. Data are represented as the mean \pm s.e.m. from one experiment with at least five mice per group. Gene expression data were log-transformed. ${ }^{*} P \leq 0.05,{ }^{* *} P<0.01,{ }^{* * *} P<0.001$. 
Administration of TUDCA Improves Disrupted Ileal Bile Acid Homeostasis in TNF ${ }^{\triangle A R E / W T}$ Mice

Because $\mathrm{TNF}^{\triangle \mathrm{ARE} / \mathrm{WT}}$ mice displayed major changes in ileal bile acid homeostasis, we used this mouse model to further study the effects of TUDCA on the expression of nuclear receptors and bile acid receptors in vivo. At 4 weeks of age, male $\mathrm{TNF}^{\triangle \mathrm{ARE} / \mathrm{WT}}$ mice and their wild-type littermates were treated with $2 \mathrm{~g} / \mathrm{l}$ TUDCA in the drinking water ad libitum

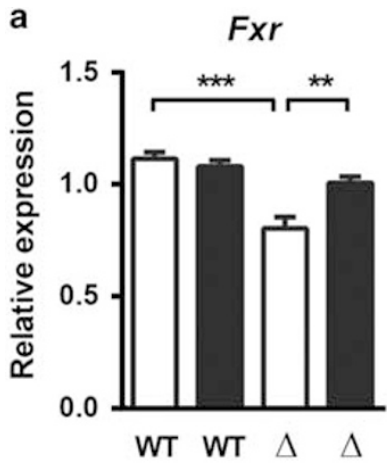

TUDCA - + - +

b

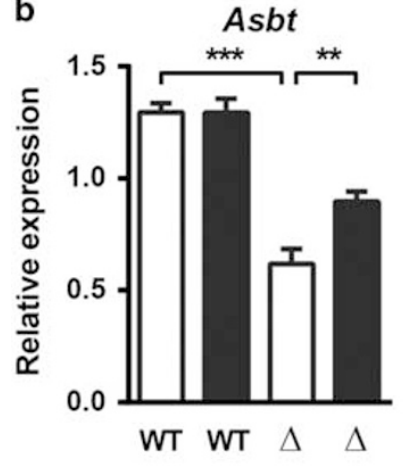

TUDCA - + - +

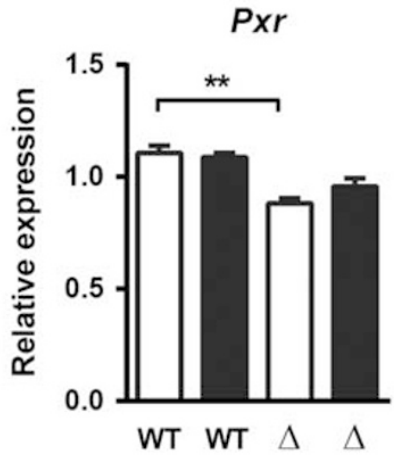

TUDCA - + - +

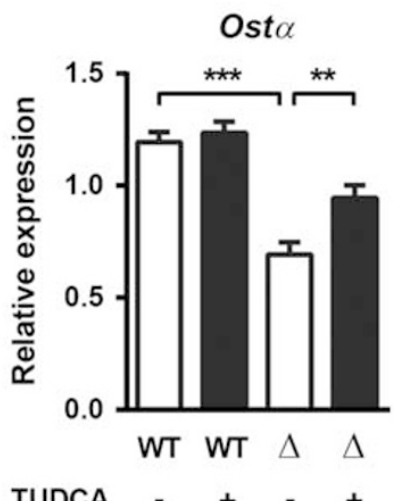

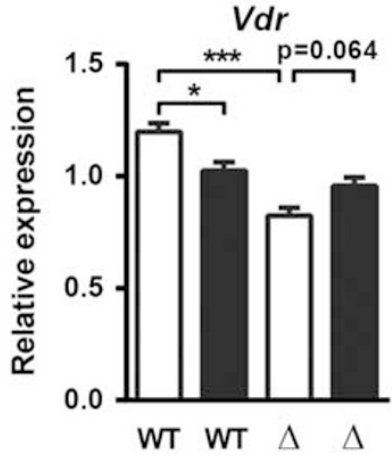

TUDCA - + - +

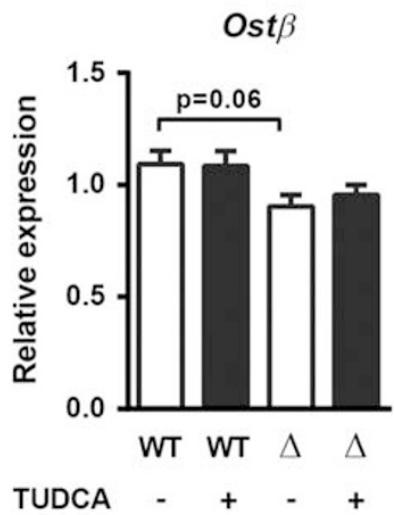

c

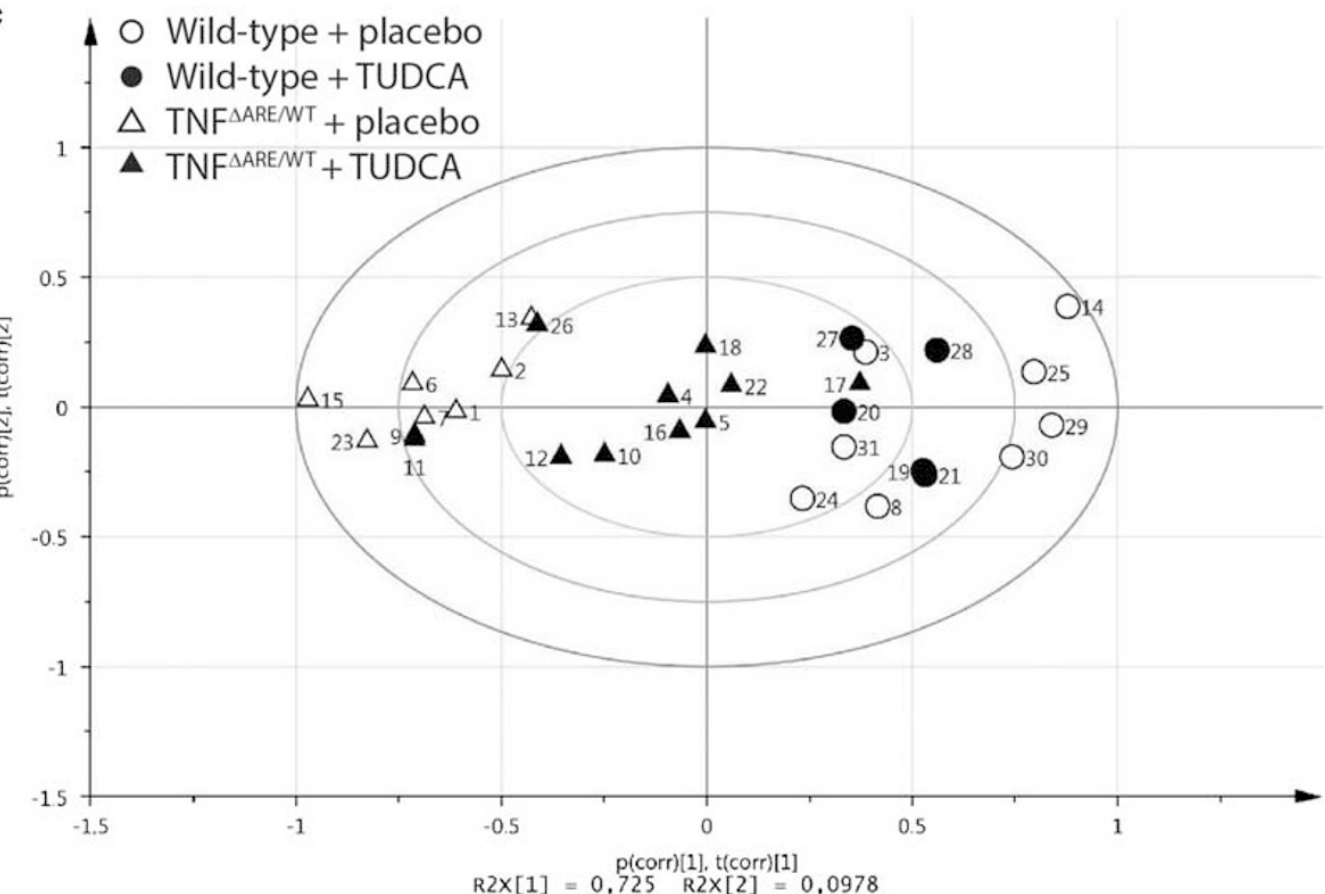



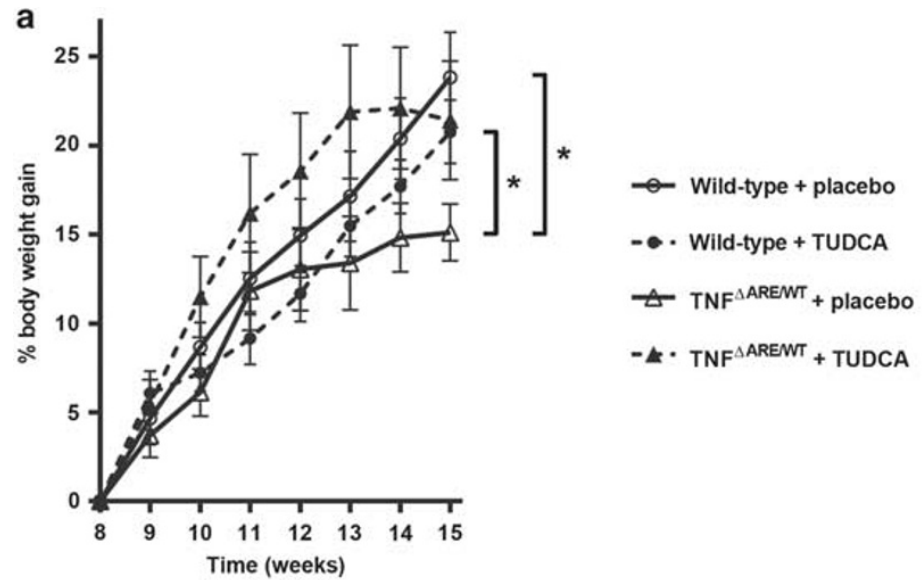

b

Wild-type + placebo
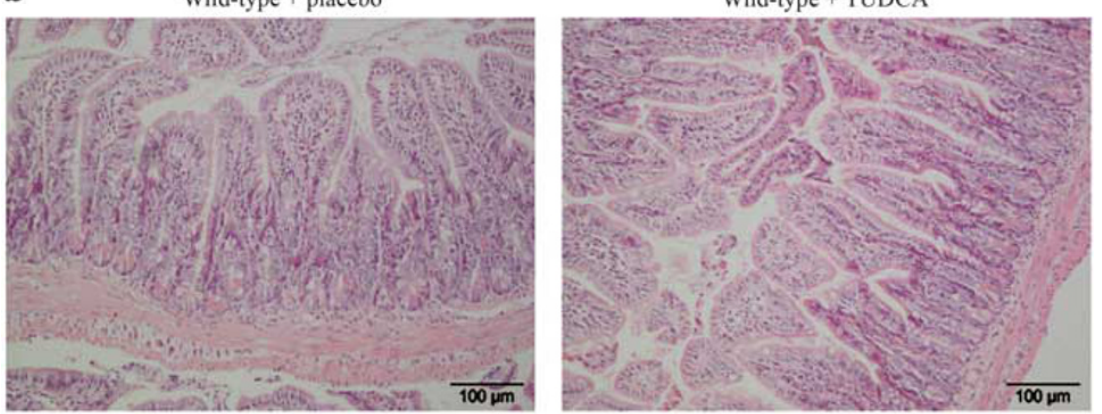

TNF $^{\triangle A R E W T}+$ placebo

TNF $\triangle A R E W T+T U D C A$
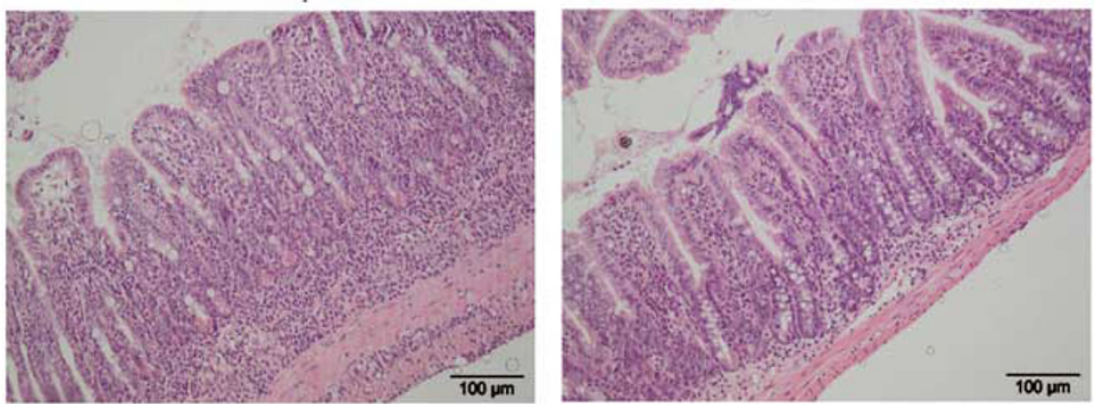

C

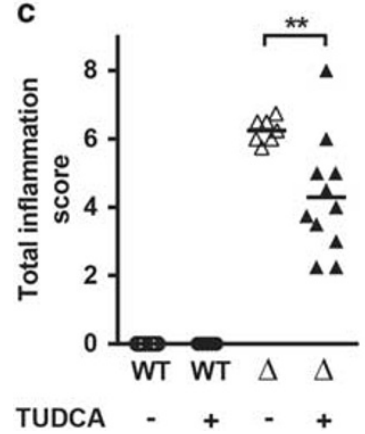

d

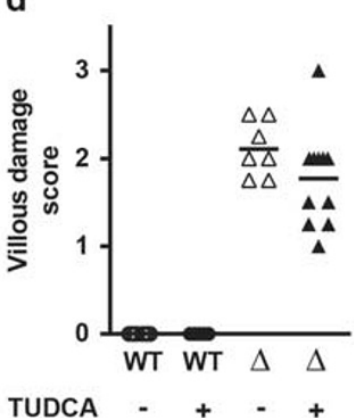

e

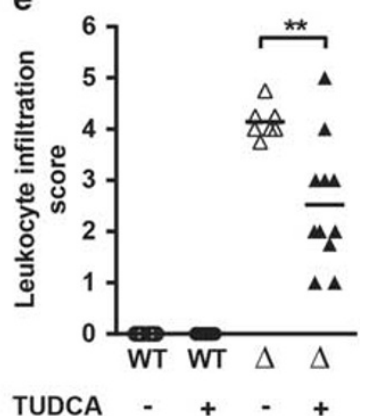

Figure 4 Effect of TUDCA administration on the severity of chronic ileitis in 15-week-old TNF $\triangle A R E / W T$ mice. (a) Progressive body weight gain starting at 8 weeks of age in placebo-treated wild-type (WT) mice $(N=8$; open circles), TUDCA-treated wild-type mice $(N=5$; filled circles), placebo-treated $\operatorname{TNF}^{\triangle A R E} / \mathrm{WT}$ mice $\left(N=7\right.$; open triangles) and TUDCA-treated TNF ${ }^{\triangle A R E} / \mathrm{WT}$ mice $(N=11$; filled triangles). (b) Representative pictures $(200 \times)$ of hematoxylin and eosin stained sections of the terminal ileum of wild-type mice and TNF ${ }^{A A R E}{ }^{-W T}$ mice treated with TUDCA or placebo, showing reduced inflammatory cell infiltration in the ileum of TNF ${ }^{\triangle A R E / W T}$ mice that were treated with TUDCA. The total histological inflammation score (c), calculated as the sum of villous damage (d) and leukocyte infiltration (e) scores. Data are represented as the mean \pm s.e.m. from one experiment with at least five mice per group. ${ }^{*} P \leq 0.05,{ }^{*} P<0.01$. Scale bars: $100 \mu \mathrm{m}$. 
(as previously described by Cao et $a .^{38}$ ) until the age of 15 weeks. Placebo-treated $\mathrm{TNF}^{\triangle \mathrm{ARE} / \mathrm{WT}}$ mice and wild-type littermates received normal drinking water. TUDCA upregulated the expression levels of $F x r$ and $V d r$ in the distal ileum of $\mathrm{TNF}^{\triangle \mathrm{ARE} / \mathrm{WT}}$ mice $(P=0.001$ and $P=0.064$ compared with placebo-treated $\mathrm{TNF}^{\triangle \mathrm{ARE} / \mathrm{WT}}$ mice, respectively), while $P x r$ expression did not change (Figure 3a). Furthermore, prolonged administration of TUDCA to these mice increased the mRNA expression of the bile acid transporter genes Asbt $(P=0.002)$ and Ost $\alpha(P=0.008)$, but not Ost $\beta(P=0.756)$ (Figure $3 \mathrm{~b}$ ). Although the administration of TUDCA to wildtype mice resulted in a downregulation of $V d r$ compared to placebo-treated wild-type mice $(P=0.017)$, baseline mRNA expression levels of all other genes analyzed remained unaffected in wild-type mice following TUDCA treatment (Figures $3 \mathrm{a}$ and $\mathrm{b}$ ). Principal component analysis was additionally performed to visualize the gene expression data of nuclear receptors and bile acid transporters. The corresponding score plot demonstrates a clear separation between placebo-treated $\mathrm{TNF}^{\triangle \mathrm{ARE} / \mathrm{WT}}$ mice and wild-type littermates (Figure 3c). Whereas TUDCA-treated and placebo-treated wild-type mice cluster together, TUDCAtreated $\mathrm{TNF}^{\triangle \mathrm{ARE} / \mathrm{WT}}$ mice shift toward the wild-type mice. The plot also shows that two TNF ${ }^{\Delta \mathrm{ARE} / \mathrm{WT}}$ mice responded poorly to the TUDCA treatment, as they cluster within the placebo-treated $\mathrm{TNF}^{\triangle \mathrm{ARE} / \mathrm{WT}}$ group. Taken together, these results correspond to the observed effects of TUDCA in Caco2 cell monolayers and suggest that TUDCA protects bile acid homeostasis under inflammatory conditions in vivo.

\section{Administration of TUDCA Attenuates Chronic lleitis in TNF $^{\triangle A R E / W T}$ Mice}

Finally, we examined whether prolonged administration of TUDCA exerted therapeutic effects in $\mathrm{TNF}^{\triangle \mathrm{ARE} / \mathrm{WT}}$ mice. From 8 weeks onward, these mice spontaneously develop ileitis similar to human $\mathrm{CD}$, with lower body weight and histological abnormalities in the distal ileum. ${ }^{46}$ The body weight of placebo-treated wild-type mice continued to increase until the end of the study, while body weight gain in placebo-treated $\mathrm{TNF}^{\triangle \mathrm{ARE} / \mathrm{WT}}$ mice started to decrease at the age of 11 weeks (Figure $4 \mathrm{a}, P=0.026$ compared with wild-type littermates). In contrast, body weight gain of $\mathrm{TNF}^{\triangle \mathrm{ARE} / \mathrm{WT}}$ mice that received TUDCA was maintained until 13 weeks of age (Figure 4a, $P=0.042$ compared with placebo-treated $T N F^{\triangle A R E / W T}$ mice). The overall body weight of TUDCA-treated wild-type mice did not differ significantly from placebo-treated wild-type mice. Histopathological evaluation of distal ileum sections from $\mathrm{TNF}^{\mathrm{ARE} / \mathrm{WT}}$ mice showed obvious signs of inflammation, characterized by distortion of the villi and severe leukocyte infiltration into the mucosa, submucosa and muscularis externa (Figure $4 b$ ). The total inflammation score was significantly lower in the TUDCA-treated $\mathrm{TNF}^{\triangle \mathrm{ARE} / \mathrm{WT}}$ group $(P=0.009$ compared with placebo-treated $T_{N F}{ }^{\triangle A R E / W T}$ mice; Figure $4 \mathrm{c}$ ). More specifically, long-term administration of TUDCA to
$\mathrm{TNF}^{\triangle \mathrm{ARE} / \mathrm{WT}}$ mice did not prevent alterations in villous architecture $(P=0.168$ compared with placebo-treated $\mathrm{TNF}^{\triangle \mathrm{ARE} / \mathrm{WT}}$ mice; Figure $4 \mathrm{~d}$ ) but reduced inflammatory cell infiltration $(P=0.004$ compared with placebo-treated $\mathrm{TNF}^{\triangle \mathrm{ARE} / \mathrm{WT}}$ mice; Figure 4e). We therefore conclude that TUDCA significantly improves murine CD-like ileitis.

\section{Discussion}

Bile acids have recently been described as signaling molecules that activate a subset of nuclear receptors including FXR, PXR and VDR. ${ }^{4}$ These receptors are key regulators of inflammation ${ }^{3}$ and increasing evidence suggests that signaling through these receptors is compromised in human IBD. ${ }^{13-22}$ In the present study, we demonstrated that TUDCA, a secondary bile acid with cytoprotective effects, ${ }^{37}$ alleviates the downregulation of nuclear receptors and bile acid transporters under inflammatory conditions, both in vitro and in vivo, and alleviates CD-like ileitis in mice.

TNF $\alpha$ is a key cytokine involved in the intestinal inflammation in human and experimental IBD. ${ }^{45,47}$ Using both an in vitro and in vivo model, we illustrated the ability of this cytokine to directly impair the expression of genes involved in ileal bile acid transport and signaling. Previous studies also showed a significant contribution of cytokines such as IL-1 $\beta$, IL- 6 and TNF $\alpha$, on the regulation of intestinal and hepatobiliary transporters and nuclear receptors. ${ }^{26-31}$

Because the TNF ${ }^{\triangle \mathrm{ARE} / \mathrm{WT}}$ mouse model is widely used as a CD model, ${ }^{45,46}$ we compared gene expression patterns of ileal bile acid transporters and nuclear receptors in $\mathrm{TNF}^{\triangle \mathrm{ARE} / \mathrm{WT}}$ mice with those observed in ileal $\mathrm{CD}$ patients with active disease. In line with previous studies, ${ }^{20,22,24,48}$ we found a consistent downregulation of FXR, PXR and VDR and the bile acid uptake transporter ASBT in active CD. Although Jahnel et al. ${ }^{20}$ published comparable results, they did not find decreased mRNA expression of PXR. The precise explanation for this discrepancy is unclear, but cannot be attributed to the location of tissue sampling because PXR is expressed at a constant level along the length of the small intestine. ${ }^{22}$ However, in the study of Jahnel et al. most patients were under concomitant therapy, whereas in our cohort, most patients were not receiving treatment as they were newly diagnosed for CD. Since gene expression levels of $P X R$ are enhanced by drugs such as corticosteroids, ${ }^{49-51}$ concomitant medication use might account for the discrepancy in outcome. Interestingly, we found that the changes in expression levels of genes involved in bile acid homeostasis in $\mathrm{TNF}^{\triangle \mathrm{ARE} / \mathrm{WT}}$ mice closely resemble the alterations observed in $\mathrm{CD}$ ileitis. Therefore, we believe that the $\mathrm{TNF}^{\triangle \mathrm{ARE} / \mathrm{WT}}$ mouse model is a suitable model to study defects in bile acid homeostasis, which are a major feature in ileal CD.

The effects of bile acids on the expression of genes involved in intestinal bile acid homeostasis have been reported previously. ${ }^{33,34,36,51-53}$ These results, however, are sometimes 
contradictory and vary between different bile acid species. In the present study, we showed that TUDCA protected against dysregulated expression of nuclear receptors and bile acid transporters in response to TNF $\alpha$. Of note, prolonged administration of TUDCA enhanced the expression of genes involved in ileal bile acid homeostasis during inflammation in $\mathrm{TNF}^{\triangle \mathrm{ARE} / \mathrm{WT}}$ mice, but not under steady-state conditions in wild-type mice. This is an important finding given the crucial role of nuclear receptors in the counter-regulation of intestinal inflammation. ${ }^{3,4}$ These observations may suggest that TUDCA improves immunomodulatory bile acid signaling through these receptors, either directly (by regulating nuclear receptor expression) or indirectly (by regulating cellular bile acid transport).

Previous studies by both our group and others have shown that either oral or intraperitoneal administration of TUDCA attenuates murine colonic inflammation elicited by dextran sodium sulfate. ${ }^{38-40}$ However, more than $95 \%$ of the intestinal bile acid pool does not move down into the colon due to efficient reabsorption in the distal ileum, ${ }^{23}$ which makes it more appropriate to consider TUDCA supplementation as a therapeutic approach for small bowel inflammation. The present study showed that early and prolonged administration of TUDCA attenuates chronic ileal inflammation, as evidenced by reduced histological inflammatory cell infiltration and improved body weight gain. To our knowledge, only one other study has previously examined the effect of TUDCA on ileal inflammation. In contrast to our results, it was reported that dietary TUDCA treatment exacerbates indomethacin-induced ileitis in rats. ${ }^{54}$ Differences in dosage are unlikely to explain the discrepancy in outcome, since the dose of TUDCA in both studies was estimated to be equivalent to $400 \mathrm{mg} / \mathrm{kg}$ body weight. However, the presence of bile acids is crucial in the pathogenesis of indomethacininduced ileal inflammation, ${ }^{55}$ making this an inferior model to study the therapeutic effects of exogenously administered bile acids in IBD.

Finally, as TUDCA was previously found to reduce cytokine responses in vitro, ${ }^{56-58}$ one could speculate that the stabilizing effects of TUDCA on the expression of bile acid transporters and nuclear receptors are secondary to antiinflammatory actions. However, in our study, TUDCA partly antagonized the TNF $\alpha$-effect on bile acid transporter and nuclear receptor expression in Caco-2 cell monolayers, but could not prevent the TNF $\alpha$-induced secretion of IL- 8 and decrement in TEER. The reason why TUDCA even further reduced TEER is unclear but cannot be related to cytotoxicity. Together with the observation that TUDCA affected the expression of genes involved in bile acid homeostasis only in the inflamed ileum, this raises the possibility that TUDCA directly regulates the expression of bile acid transporters and nuclear receptors during inflammatory conditions which in turn could be a crucial mechanism by which an antiinflammatory effect is obtained in the longer term. Further studies are required to address this hypothesis.
In summary, we showed that TUDCA alleviates the disruption of bile acid homeostasis upon TNF $\alpha$ stimulation both in vitro and in vivo. In addition, we demonstrated that early and prolonged administration of TUDCA dampens $\mathrm{CD}$-like ileitis in mice. Together with earlier promising results in experimental colitis, ${ }^{38-40}$ we strongly believe that TUDCA should be investigated as a therapeutic agent in patients with IBD.

Supplementary Information accompanies the paper on the Laboratory Investigation website (http://www.laboratoryinvestigation.org)

\section{ACKNOWLEDGMENTS}

We thank Dr G. Kollias (Alexander Fleming Biomedical Sciences Research Center, Vari, Greece) for providing the TNF ${ }^{\triangle A R E N T}$ mice and Dr Falk

Pharmaceuticals, who kindly provided TUDCA for research purposes. We also thank Elien Glorieus for the generous help in obtaining patient samples and Hilde Devlies, Griet Driesschaert, and Petra Van Wassenhove for offering technical assistance. This work was supported by research grants from the Research Foundation Flanders (FWO; 11 J9915N and 1298213N) and a grant from the Belgian foundation for Crohn's disease and ulcerative colitis patients (CCV vzw, research grant 2014).

\section{DISCLOSURE/CONFLICT OF INTEREST}

The authors declare no conflict of interest.

1. Cammarota G, laniro G, Cianci $R$, et al. The involvement of gut microbiota in inflammatory bowel disease pathogenesis: potential for therapy. Pharmacol Ther 2015;149:191-212.

2. Duboc H, Rajca S, Rainteau D, et al. Connecting dysbiosis, bile-acid dysmetabolism and gut inflammation in inflammatory bowel diseases. Gut 2013;62:531-539.

3. Fiorucci S, Cipriani S, Mencarelli A, et al. Counter-regulatory role of bile acid activated receptors in immunity and inflammation. Curr Mol Med 2010;10:579-595.

4. Copple BL, Li T. Pharmacology of bile acid receptors: evolution of bile acids from simple detergents to complex signaling molecules. Pharmacol Res 2015;104:9-21.

5. Vavassori $P$, Mencarelli $A$, Renga $B$, et al. The bile acid receptor FXR is a modulator of intestinal innate immunity. J Immunol 2009;183:6251-6261.

6. Gadaleta RM, Erpecum KJ, van, Oldenburg B, et al. Farnesoid X receptor activation inhibits inflammation and preserves the intestinal barrier in inflammatory bowel disease. Gut 2011;60:463-472.

7. Sun J, Mustafi R, Cerda $S$, et al. Lithocholic acid down-regulation of NF-KB activity through vitamin D receptor in colonic cancer cells. J Steroid Biochem Mol Biol 2008;111:37-40.

8. Shah YM, Ma X, Morimura K, et al. Pregnane $\mathrm{X}$ receptor activation ameliorates DSS-induced inflammatory bowel disease via inhibition of NF-kB target gene expression. Am J Physiol Gastrointest Liver Physiol 2007;292:G1114-G1122.

9. Froicu M, Cantorna MT. Vitamin D and the vitamin D receptor are critical for control of the innate immune response to colonic injury. BMC Immunol 2007;8:5.

10. Venkatesh M, Mukherjee S, Wang H, et al. Symbiotic bacterial metabolites regulate gastrointestinal barrier function via the xenobiotic sensor PXR and toll-like receptor 4. Immunity 2014;41:296-310.

11. Inagaki $T$, Moschetta $A$, Lee $Y-K$, et al. Regulation of antibacterial defense in the small intestine by the nuclear bile acid receptor. Proc Natl Acad Sci USA 2006;103:3920-3925.

12. Zhou C. Mutual repression between steroid and xenobiotic receptor and NF-kB signaling pathways links xenobiotic metabolism and inflammation. J Clin Invest 2006;116:2280-2289.

13. Dring $\mathrm{MM}$, Goulding $\mathrm{CA}$, Trimble $\mathrm{VI}$, et al. The pregnane $\mathrm{X}$ receptor locus is associated with susceptibility to inflammatory bowel disease. Gastroenterology 2006;130:341-348. 
14. Attinkara R, Mwinyi J, Truninger $\mathrm{K}$, et al. Association of genetic variation in the $\mathrm{NR} 1 \mathrm{H} 4$ gene, encoding the nuclear bile acid receptor FXR, with inflammatory bowel disease. BMC Res Notes 2012;5:461.

15. Naderi N, Farnood A, Habibi M, et al. Association of vitamin D receptor gene polymorphisms in Iranian patients with inflammatory bowel disease. J Gastroenterol Hepatol 2008;23:1816-1822.

16. Simmons JD, Mullighan $\mathrm{C}$, Welsh $\mathrm{Kl}$, et al. Vitamin $\mathrm{D}$ receptor gene polymorphism: association with Crohn's disease susceptibility. Gut 2000;47:211-214.

17. Hughes DJ, McManus $R$, Neary $P$, et al. Common variation in the vitamin $\mathrm{D}$ receptor gene and risk of inflammatory bowel disease in an Irish case-control study. Eur J Gastroenterol Hepatol 2011;23:807-812.

18. Wang L, Wang ZT, Hu JJ, et al. Polymorphisms of the vitamin D receptor gene and the risk of inflammatory bowel disease: a metaanalysis. Genet Mol Res 2014;13:2598-2610.

19. Glas J, Seiderer J, Fischer D, et al. Pregnane X receptor (PXR/NR1/2) gene haplotypes modulate susceptibility to inflammatory bowel disease. Inflamm Bowel Dis 2011;17:1917-1924.

20. Jahnel J, Fickert $P$, Hauer $A C$, et al. Inflammatory bowel disease alters intestinal bile acid transporter expression. Drug Metab Dispos Biol Fate Chem 2014;42:1423-1431.

21. Langmann $T$, Moehle $C$, Mauerer $R$, et al. Loss of detoxification in inflammatory bowel disease: dysregulation of pregnane $\mathrm{X}$ receptor target genes. Gastroenterology 2004;127:26-40.

22. Shakhnovich V, Vyhlidal CA, Friesen $C$, et al. Decreased pregnane $X$ receptor (PXR) expression in children with active Crohn's disease. Drug Metab Dispos 2016;44:1066-1069.

23. Dawson PA, Karpen SJ. Intestinal transport and metabolism of bile acids. J Lipid Res 2015;56:1085-1099.

24. Wojtal KA, Eloranta JJ, Hruz $\mathrm{P}$, et al. Changes in mRNA expression levels of solute carrier transporters in inflammatory bowel disease patients. Drug Metab Dispos Biol Fate Chem 2009;37:1871-1877.

25. Ballatori N, Fang F, Christian WV, et al. Osta-Ost $\beta$ is required for bile acid and conjugated steroid disposition in the intestine, kidney, and liver. Am J Physiol Gastrointest Liver Physiol 2008;295:G179-G186.

26. Kim MS, Shigenaga J, Moser $A$, et al. Repression of farnesoid $X$ receptor during the acute phase response. J Biol Chem 2003;278:8988-8995.

27. Teng $\mathrm{S}$, Piquette-Miller $\mathrm{M}$. The involvement of the pregnane $\mathrm{X}$ receptor in hepatic gene regulation during inflammation in mice. J Pharmacol Exp Ther 2005;312:841-848.

28. Geier A, Dietrich CG, Voigt S, et al. Cytokine-dependent regulation of hepatic organic anion transporter gene transactivators in mouse liver. Am J Physiol Gastrointest Liver Physiol 2005;289:G831-G841.

29. Neimark E, Chen F, Li X, et al. c-Fos is a critical mediator of inflammatory-mediated repression of the apical sodium-dependent bile acid transporter. Gastroenterology 2006;131:554-567.

30. Chen F, Ma L, Sartor RB, et al. Inflammatory-mediated repression of the rat ileal sodium-dependent bile acid transporter by c-fos nuclear translocation. Gastroenterology 2002;123:2005-2016.

31. Merrell MD, Nyagode BA, Clarke JD, et al. Selective and cytokinedependent regulation of hepatic transporters and bile acid homeostasis during infectious colitis in mice. Drug Metab Dispos Biol Fate Chem 2014;42:596-602.

32. Zollner $G$, Fickert $P$, Fuchsbichler $A$, et al. Role of nuclear bile acid receptor, FXR, in adaptive $A B C$ transporter regulation by cholic and ursodeoxycholic acid in mouse liver, kidney and intestine. J Hepatol 2003:39:480-488.

33. Zollner $G$, Wagner M, Moustafa $T$, et al. Coordinated induction of bile acid detoxification and alternative elimination in mice: role of FXRregulated organic solute transporter- $\alpha / \beta$ in the adaptive response to bile acids. Am J Physiol Gastrointest Liver Physiol 2006;290:G923-G932.

34. Song P, Rockwell CE, Cui JY, et al. Individual bile acids have differential effects on bile acid signaling in mice. Toxicol Appl Pharmacol 2015;283:57-64.

35. Duane WC, Xiong W, Wolvers J. Effects of bile acids on expression of the human apical sodium dependent bile acid transporter gene. Biochim Biophys Acta 2007;1771:1380-1388.

36. Stravitz RT, Sanyal AJ, Pandak WM, et al. Induction of sodiumdependent bile acid transporter messenger RNA, protein, and activity in rat ileum by cholic acid. Gastroenterology 1997;113:1599-1608.
37. Amaral JD, Viana RJS, Ramalho RM, et al. Bile acids: regulation of apoptosis by ursodeoxycholic acid. J Lipid Res 2009;50:1721-1734.

38. Cao SS, Zimmermann EM, Chuang B-M, et al. The unfolded protein response and chemical chaperones reduce protein misfolding and colitis in mice. Gastroenterology 2013;144:989-1000, e6.

39. Laukens D, Devisscher L, Van den Bossche L, et al. Tauroursodeoxycholic acid inhibits experimental colitis by preventing early intestinal epithelial cell death. Lab Investig J Tech Methods Pathol 2014;94:1419-1430.

40. Hino K, Saito A, Asada R, et al. Increased susceptibility to dextran sulfate sodium-induced colitis in the endoplasmic reticulum stress transducer OASIS deficient mice. PloS One 2014;9:e88048.

41. Son DO, Satsu H, Shimizu M. Histidine inhibits oxidative stress- and TNF-a-induced interleukin-8 secretion in intestinal epithelial cells. FEBS Lett 2005;579:4671-4677.

42. Ren $\mathrm{D}-\mathrm{Y}, \mathrm{Li} \mathrm{C}$, Qin $\mathrm{Y}-\mathrm{Q}$, et al. Lactobacilli reduce chemokine IL-8 production in response to TNF-a and salmonella challenge of Caco-2 cells. BioMed Res Int 2013;2013:e925219.

43. Fukuda $\mathrm{T}$, Majumder $\mathrm{K}$, Zhang $\mathrm{H}$, et al. Adenine inhibits TNF-a signaling in intestinal epithelial cells and reduces mucosal inflammation in a dextran sodium sulfate-induced colitis mouse model. J Agric Food Chem 2016;64:4227-4234.

44. Ma TY, Iwamoto GK, Hoa NT, et al. TNF-a-induced increase in intestinal epithelial tight junction permeability requires NF-KB activation. Am J Physiol Gastrointest Liver Physiol 2004;286:G367-G376.

45. Kontoyiannis $D$, Pasparakis $M$, Pizarro $\Pi$, et al. Impaired on/off regulation of TNF biosynthesis in mice lacking TNF AU-rich elements: implications for joint and gut-associated immunopathologies. Immunity 1999;10:387-398.

46. Baur $\mathrm{P}$, Martin F-P, Gruber $\mathrm{L}$, et al. Metabolic phenotyping of the Crohn's disease-like IBD etiopathology in the TNF( $\triangle$ ARE/WT) mouse model. J Proteome Res 2011;10:5523-5535.

47. Soufli I, Toumi R, Rafa $\mathrm{H}$, et al. Overview of cytokines and nitric oxide involvement in immuno-pathogenesis of inflammatory bowel diseases. World J Gastrointest Pharmacol Ther 2016;7: 353-360.

48. Jung $D$, Fantin $A C$, Scheurer $U$, et al. Human ileal bile acid transporter gene ASBT (SLC10A2) is transactivated by the glucocorticoid receptor. Gut 2004;53:78-84.

49. Shi D, Yang D, Yan B. Dexamethasone transcriptionally increases the expression of the pregnane $X$ receptor and synergistically enhances pyrethroid esfenvalerate in the induction of cytochrome P450 $3 \mathrm{~A} 23$. Biochem Pharmacol 2010;80:1274-1283.

50. Pascussi JM, Drocourt L, Fabre JM, et al. Dexamethasone induces pregnane $\mathrm{X}$ receptor and retinoid $\mathrm{X}$ receptor-alpha expression in human hepatocytes: synergistic increase of CYP3A4 induction by pregnane $X$ receptor activators. Mol Pharmacol 2000;58:361-372.

51. Khan AA, Chow ECY, van Loenen-Weemaes AM, et al. Comparison of effects of VDR versus PXR, FXR and GR ligands on the regulation of CYP3A isozymes in rat and human intestine and liver. Eur J Pharm Sci Off J Eur Fed Pharm Sci 2009;37:115-125.

52. Frankenberg $T$, Rao $A$, Chen $F$, et al. Regulation of the mouse organic solute transporter alpha-beta, Ostalpha-Ostbeta, by bile acids. Am J Physiol Gastrointest Liver Physiol 2006;290:G912-G922.

53. Khan AA, Chow ECY, Porte RJ, et al. Expression and regulation of the bile acid transporter, OSTalpha-OSTbeta in rat and human intestine and liver. Biopharm Drug Dispos 2009;30:241-258.

54. Uchida A, Yamada T, Hayakawa T, et al. Taurochenodeoxycholic acid ameliorates and ursodeoxycholic acid exacerbates small intestinal inflammation. Am J Physiol 1997;272:G1249-G1257.

55. Brodie DA, Cook PG, Bauer BJ, et al. Indomethacin-induced intestinal lesions in the rat. Toxicol Appl Pharmacol 1970;17:615-624.

56. Saitoh O, Nakagawa K, Sugi K, et al. Bile acids inhibit tumour necrosis factor alpha-induced interleukin-8 production in human colon epithelial cells. J Gastroenterol Hepatol 1998;13:1212-1217.

57. Neuman MG, Shear NH, Bellentani S, et al. Role of cytokines in ethanol-induced cytotoxicity in vitro in Hep G2 cells. Gastroenterology 1998;115:157-166.

58. Li $\mathrm{Y}$, Zhang $\mathrm{H}$, Jiang $\mathrm{C}$, et al. Hyperhomocysteinemia promotes insulin resistance by inducing endoplasmic reticulum stress in adipose tissue. J Biol Chem 2013:288:9583-9592. 